\title{
Study on Polysaccharide by the Fluorescence Method. I. Fluorescence Depolarization of the Fluorescein Conjugates of Amylose and Dextran
}

\author{
Shinichi Kitamura, Harunobu YunoKawa, and Takashi KugE \\ Laboratory of Biopolymers, Department of Agricultural Chemistry, \\ Kyoto Prefectural University, Sakyo-ku, Kyoto 606, Japan.
}

(Received March 23, 1981)

\begin{abstract}
Amylose and dextran conjugated with fluorescein were prepared and the degree of fluorescence polarization of these conjugates was measured with changing solvent viscosity at constant temperature $\left(25^{\circ} \mathrm{C}\right)$. The results obtained were analyzed by Perrin's equation to calculate the mean rotational relaxation time. The value for dextran conjugate was very small compared with that for amylose. This suggests that the molecular chain of dextran is more flexible than that of amylose. Polarizations of these conjugates gradually departed from the Perrin equation when the temperature was raised in a given solvent. This discrepancy can be attributed to an accelerated loosening of the internal structure of these polysaccharide chains. Thus it seems that amylose and dextran molecules below the transition temperatures have partly ordered conformations with restricted rotation about glycosidic linkage.
\end{abstract}

KEY WORDS Polysaccharide / Amylose / Dextran / Fluorescence Polarization / Fluorescein Conjugate / Segmental Motion / Flexibility / Conformational Transition /

The fluorescence polarization method has been used to study rotational diffusion of macromolecules in solution. ${ }^{1,2}$ Many studies on fluorescent conjugates of flexible polymers have been reported, and show that this method is useful for obtaining information on the segmental motion and conformation of these polymers. ${ }^{3-10}$ This paper describes the application of this method to polysaccharides exhibiting conformational transitions.

A polysaccharide chain contains a large number of glycoside links which allow the glucose units to rotate about them with a relatively small change in internal energy. Thus, the molecular chain in solution can fluctuate among various possible conformations. ${ }^{11}$ In this sense, a polysaccharide chain is flexible. However, the rotation about the glycoside links is more or less restricted by non-bonded interactions between glucose units, and the segmental motion of the polymer chain may also be restricted to some extent.

One of our purposes in the present study has been to obtain information on the segmental motion in polysaccharide chains by the fluorescence polar- ization method. For this purpose we chose two polysaccharides, amylose and dextran. Both consist of the same kind of monomer units but are jointed with different linkages: the $\alpha$-1,4-glucoside linkage for amylose and the $\alpha$-1,6-glucoside linkage for dextran. The latter has one extra single rotatory bond per monomer unit compared with the former. Conformational energy calculations with nonbonded interactions suggest that dextran may be more flexible than amylose. ${ }^{12,13}$ We tested the validity of this suggestion by using the fluorescence polarization method on these polysaccharides.

Amylose is considered to undergo a thermallyinduced conformational transition, i.e., a helix-coil transition in solution. ${ }^{14}$ Thus we investigated the effects of temperature on the segmental motion of amylose and dextran in order to detect such conformational transitions.

\section{EXPERIMENTAL}

\section{Materials}

The amylose used was "AVEBE amylose," pro- 
vided by Nichiden Kagaku Co., Ltd., Osaka. This material was purified by precipitating it several times with butanol from its aqueous solution. The viscosity-average molecular weight, $M_{v}$, was found to be $3.89 \times 10^{5}$ from viscosity measurernents in dimethyl sulfoxide (DMSO) using eq $1 .^{15}$

$$
[\eta]=8.1 \times 10^{-4} M^{0.91}
$$

Dextran was purchased from Pharmacia Chemicals Co., Ltd. and used without further purification. The weight-average molecular weight was $4.66 \times 10^{5}$, a value provided by the Pharmacia Chemical Co., Ltd. Fluorescein isothiocyanate was purchased from B.B.L., Division of Bio. Quest. $\beta$-Cyclodextrin used was that previously prepared and its purity was checked by TLC at this laboratory. ${ }^{16}$ Other chemicals were of reagent grade unless otherwise specified.

\section{Preparation of Fluorescent Conjugates}

DMSO was used after vacuum distillation. Pyridine was distilled in the presence of calcium hydride before use. Conjugation was carried out according to the method of Belder. ${ }^{17}$ The carbohydrates $(500 \mathrm{mg})$ were dissolved in DMSO $(5 \mathrm{ml})$ containing a few drops of pyridine. Fluorescein isothiocyanate $(25 \mathrm{mg})$ was added, followed by the addition of dibutyltin dilaurate, and the mixture was heated for $2 \mathrm{~h}$ at $95^{\circ} \mathrm{C}$. Care was taken to avoid moisture. The mixture was then poured in ethanol to precipitate the conjugate produced, filtered off and dried in vacuo at room temperature. It is considered that fluorescein isothiocyanate residues were bound randomly to the hydroxyl groups of the carbohydrates by this procedure.

\section{Preparation of Measured Solution}

A polysaccharide conjugate (about $8 \mathrm{mg}$ ) was dissolved in $0.5 \mathrm{ml}$ DMSO and was diluted to $20 \mathrm{ml}$ with distilled water. When used, this solution was usually diluted twenty-fold with a phosphate buffer solution of $\mathrm{pH}$ 7. Dye, carbohydrate and phosphate concentrations in this solution in the final stage were about $10^{-6} \mathrm{M}, 0.002 \%$ and $0.01 \mathrm{M}$, respectively. The solution was used immediately after preparation.

\section{Fluorescence Lifetime Measurement}

The time decay of fluorescence intensity was measured using the Ortec (9200) single-photon- counting nanosecond fluorometer. ${ }^{18}$ Fluorescence lifetimes were calculated by the curve fitting analysis using a computer (Kyoto University, FACOM-M 200).

\section{Fluorescence Measurement and Calculation of Mean Rotational Relaxation Time}

Fluorescence polarization measurements were made on a Shimadzu spectrofluorometer (type RF502) equipped with a pair of polarization filters as the polarizer and analyzer. The excitation and emission wavelengths were $450 \mathrm{~nm}$ and $520 \mathrm{~nm}$, respectively. The temperature of the solution in the cell was kept constant by a jacket thermostatically controlled to within an accuracy of $\pm 0.1^{\circ} \mathrm{C}$. The temperature was monitored by a calibrated thermistor probe immersed in the solution.

The degree of polarization, $P$, was calculated from,

$$
P=\frac{I_{\mathrm{V}}-I_{\mathrm{H}} G}{I_{\mathrm{V}}+I_{\mathrm{H}} G}
$$

where $I_{\mathrm{V}}$ and $I_{\mathrm{H}}$ represent the intensities of vertically and horizontally polarized emission with vertically polarized incident light. $G$ is the grating correction factor defined as $I_{\mathrm{V}} / I_{\mathrm{H}}$ with the horizontally polarized incident light. ${ }^{19}$ By this apparatus, the polarization could be reproduced within an error of less than a few percent. The results were analyzed by an equation of the Perrin type, ${ }^{5,20}$

$$
\frac{1}{P}-\frac{1}{3}=\left(\frac{1}{P_{0}}-\frac{1}{3}\right)\left(1+\frac{3 \tau}{\langle\rho\rangle}\right)
$$

where $P_{0}$ is the limiting polarization observed when depolarization due to the Brownian motion is absent, $\tau$ is the fluorescence lifetime and $\langle\rho\rangle$ is the mean rotational relaxation time of kinetic unit carrying the emission oscillator. When $\langle\rho\rangle$ is a linear function of $T / \eta$, eq 3 can be rewritten,

$$
\frac{1}{P}-\frac{1}{3}=\left(\frac{1}{P_{0}}-\frac{1}{3}\right)\left(1+\frac{\beta T}{\eta}\right)
$$

where $T$ is the absolute temperature, $\eta$ is the solvent viscosity and $\beta$ is a constant characteristic of a given polymer. Hence, the value of $P_{0}$ is determined by the linear extrapolation of $1 / P$ as a function of $T / \eta$ to $T / \eta=0$. The value of $T / \eta$ varied by the addition of sucrose or by changing the temperature. The sucrose solutions were prepared using a $0.01 \mathrm{M}$ phosphate buffer solution as the solvent. The vis- 
Polysaccharide Study by the Fluorescence Method. I.

Table I. Properties of fluorescein conjugates of carbohydrates ${ }^{\mathbf{a}}$

\begin{tabular}{lcccc}
\hline \multicolumn{1}{c}{ Conjugate } & Molecular & & \multicolumn{2}{c}{ Wavelength/nm } \\
\cline { 3 - 4 } & weight & DS & Absorption & $\begin{array}{c}\text { Emission } \\
\text { maximum }\end{array}$ \\
\hline Fluorescein & 376 & - & 491 & 513 \\
F-Amylose & 389000 & 0.0083 & 495 & 518 \\
F-Dextran & 466000 & 0.0083 & 494 & 518 \\
F- $\beta$-Cyclodextrin & 1134 & 0.0040 & 492 & 516 \\
\hline
\end{tabular}

a F-Amylose, F-Dextran, and F- $\beta$-Cyclodextrin represent fluorescein conjugates of amylose, dextran, and $\beta$ cyclodextrin, respectively.

DS, degree of substitution with dye is defined as the fraction of glucose residues substituted. Absorption and emission spectra were measured in $0.01 \mathrm{M}$ phosphate buffer solution of $\mathrm{pH} 7$.

cosity of the $0.01 \mathrm{M}$ phosphate buffer solution was taken to be that of water at the same temperature. The viscosities of the water and sucrose solutions were taken from International Critical Tables ${ }^{21}$ and Kagaku Binran. ${ }^{22}$

\section{RESULTS}

\section{Characterization of Amylose and Dextran Con- jugates}

The absorption spectra of both amylose and dextran conjugates were similar to that of fluorescein except for a red shift from 2 to $4 \mathrm{~nm}$. Their emission spectra were also similar to that of fluorescein except for a red shift of about $5 \mathrm{~nm}$. The degree of substitution with dye was estimated spectrophotometrically, using methyl $N$-fluoresceinylthiocarbamate as a reference. The degrees of substitution for these conjugates were small enough to allow them to behave as unmodified amylose and dextran. These results are summarized in Table I. It is interesting to compare the depolarization characteristics of the amylose and dextran conjugates, since they are similar in molecular weight, degree of substitution, and absorption and emission properties.

\section{Fluorescence Lifetimes of Amylose and Dextran Conjugates}

In order to analyze fluorescence polarization data the fluorescence lifetime, $\tau$, of the conjugated dye residue under the solvent conditions concerned should be known in advance. The necessary data were obtained from direct measurements with a nanosecond fluorometer. Figure 1 shows typical

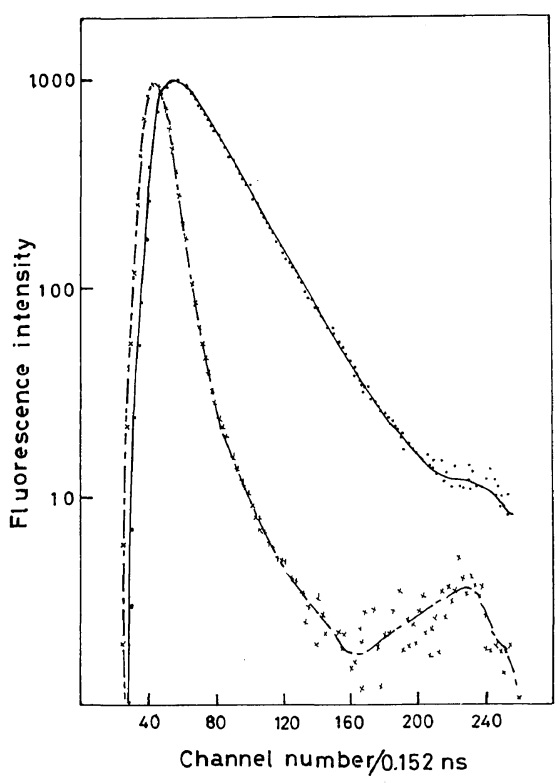

Figure 1. The fluorescence decay $(\cdots)$ for amylose conjugate in $0.01 \mathrm{M}$ phosphate buffer solution of $\mathrm{pH} 7$ at $25^{\circ} \mathrm{C}$. The decay is fitted with the theoretical curve (-) containing a single exponential decay term $(\tau=4.3)$. The sharp peak (- - ) represents the lamp profile.

decay curve of fluorescent intensity for amylose and dextran conjugates. For either conjugate, the decay curve can be represented quite well by a single exponential function, which allows $\tau$ to be determined accurately. The results are summarized in Table II. The $\tau$ values for the amylose conjugate are slightly larger than those for the dextran conjugate. However, those for each conjugate under different conditions are the same within experimental error. 
Table II. Fluorescence lifetimes of fluorescein conjugates of carbohydrates ${ }^{\mathrm{a}}$

\begin{tabular}{|c|c|c|c|}
\hline \multirow{2}{*}{ Conjugate } & \multirow{2}{*}{ Solvent } & \multirow{2}{*}{$\frac{\text { Temp }}{{ }^{\circ} \mathrm{C}}$} & \multirow{2}{*}{$\begin{array}{c}\tau \\
\mathrm{ns}\end{array}$} \\
\hline & & & \\
\hline \multirow[t]{4}{*}{ F-Amylose } & $0.01 \mathrm{M}$ Phosphate & 25 & 4.3 \\
\hline & $0.01 \mathrm{M}$ Phosphate & 40 & 4.2 \\
\hline & 0.01M Phosphate & 53 & 4.2 \\
\hline & $\begin{array}{l}0.01 \mathrm{M} \text { Phosphate, } \\
44.4 \% \text { Sucrose }\end{array}$ & 25 & 4.2 \\
\hline \multirow[t]{3}{*}{ F-Dextran } & 0.01M Phosphate & 25 & 3.9 \\
\hline & $0.01 \mathrm{M}$ Phosphate & 40 & 3.8 \\
\hline & $\begin{array}{l}0.01 \mathrm{M} \text { Phosphate, } \\
44.4 \% \text { Sucrose }\end{array}$ & 25 & 3.9 \\
\hline $\begin{array}{l}\text { F- } \beta \text {-Cyclo- } \\
\text { dextrin }\end{array}$ & $0.01 \mathrm{M}$ Phosphate & 25 & 4.0 \\
\hline
\end{tabular}

a Fluorescence lifetimes, $\tau$, were analyzed on the assumption of one component.

\section{Fluorescence Depolarization of Amylose and Dextran Conjugates}

Fluorescence polarization measurements were carried out at $25^{\circ} \mathrm{C}$ for amylose and dextran conjugates dissolved in an aqueous sucrose solution at different concentrations. Figure 2 shows Perrin plots constructed from the data obtained. For either conjugate, the plot is linear for $T / \eta$ greater than $0.5 \times 10^{4} \mathrm{~K}$ poise $^{-1}$ but shows a downward curvature at $T / \eta$ lower than that. The downward curvature in a Perrin plot suggests the presence of multiple kinetic units of largely different relaxation times. The rotation of a smaller kinetic unit may arise from the rotation of the dye itself about the bond joining it to the polysaccharide chain. This rotation may be considered to be superimposed on the slower rotation arising from the polymer chain. For such a case, Weber and Wahl have derived the following equation, ${ }^{20,23,24}$

$$
\left(\frac{1}{P}-\frac{1}{3}\right) \approx\left(\frac{1}{P_{0}^{\prime}}-\frac{1}{3}\right)\left(1+\frac{3 \tau}{\langle\rho\rangle}\right)
$$

where $1 / P_{0}{ }^{\prime}$ is the ordinate interecept obtained by extrapolating the linear part of the plot at a large value of $T / \eta$. The value of $\tau /\langle\rho\rangle$ can be calculated by eq 5 . Combining this with $\tau$, we can compute $\langle\rho\rangle$. The values of $\tau$ used for the computation are $4.3 \mathrm{~ns}$ and $3.9 \mathrm{~ns}$ for the amylose and dextran conjugates, respectively. The $\langle\rho\rangle$ thus calculated re-

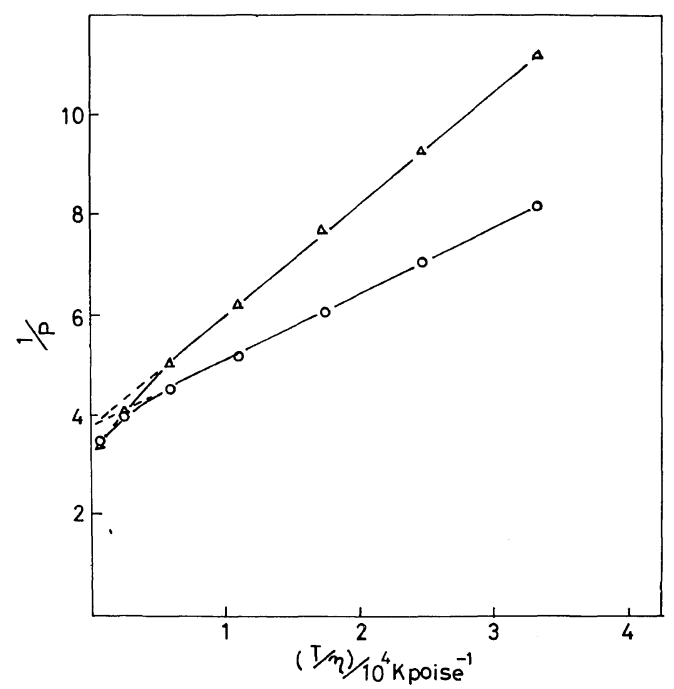

Figure 2. Plots of $1 / P$ vs. $T / \eta$ for amylose $(O)$ and dextran $(\triangle)$ conjugates in $0.01 M$ phosphate buffer solution of $\mathrm{pH} 7$ at $25^{\circ} \mathrm{C}$. Viscosity was varied by the addition of sucrose.

Table III. Comparison of $1 / P_{0}{ }^{\prime},\langle\rho\rangle$ and $T_{\mathrm{t}}$ for amylose and dextran conjugates ${ }^{\mathrm{a}}$

\begin{tabular}{lcccc}
\hline Conjugate & $1 / P_{0}{ }^{\prime}$ & \multicolumn{2}{c}{$\langle\rho\rangle$} & \multicolumn{2}{c}{$T_{\mathrm{t}}{ }^{\circ} \mathrm{C}$} \\
\cline { 5 - 6 } \cline { 5 - 5 } & & ns & $\begin{array}{l}\text { Without } \\
\text { sucrose }\end{array}$ & $\begin{array}{c}\text { With } \\
\text { sucrose }\end{array}$ \\
\hline F-Amylose & 3.9 & 11 & $46( \pm 2)^{\mathrm{b}}$ & 44 \\
F-Dextran & 3.7 & 5.4 & $26( \pm 2)^{\mathrm{b}}$ & 25 \\
\hline
\end{tabular}

a $\langle\rho\rangle$, rotational relaxation time measured at $25^{\circ} \mathrm{C}$ in $0.01 \mathrm{M}$ phosphate buffer solution of $\mathrm{pH}$ 7. $T_{t}$, transition temperature determined graphically as the point at which the experimental relation deviates from Perrin equation.

b Results are shown by mean ( \pm standard deviation) of four independent experiments.

flects the rotation due only to the larger kinetic unit, i.e., the motion of the polymer chain. The results obtained are given in Table III.

The theoretical mean relaxation time for the whole molecule of either amylose or dextran used at $25^{\circ} \mathrm{C}$ in water was estimated to be about $10^{-5} \mathrm{~s}$ from the equation given by Kirkwood and Riseman for randomly coiled polymer molecules in dilute solution. ${ }^{25}$ Since this theoretical value is much larger than the $\langle\rho\rangle$ values obtained, $\langle\rho\rangle$ may be 
associated with micro-Brownian motion of a polymer segment. The value for the dextran conjugate is much smaller than that for the amylose conjugate. This fact indicates that the dextran chain undergoes rapid micro-Brownian motion compared with amylose.

\section{Effect of Temperature on the Polarization}

In polarization measurements, $T / \eta$ also varied by changing the temperature of the solution. If $\langle\rho\rangle$ depends only on the ratio $T / \eta$, Perrin plots for data obtained at various concentrations of sucrose and at various temperatures should show the same behavior. Typical Perrin plots constructed by changing temperature are shown in Figure 3. It is seen that either plot is a straight line at low values of $T / \eta$ but changes to a continuous upward curve at higher $T / \eta$ values. The transition temperature, $T_{t}$, shown in Table III was determined graphically as the point at which the experimental relation deviates from linearity. The slopes of the straight line and $P_{0}$ obtained are greater than those for the case in which $T / \eta$ changes by the addition of sucrose. From the linear portions of these plots, $\langle\rho\rangle$ for amylose and dextran conjugates at $25^{\circ} \mathrm{C}$ are calculated to be $7.2 \mathrm{~ns}$

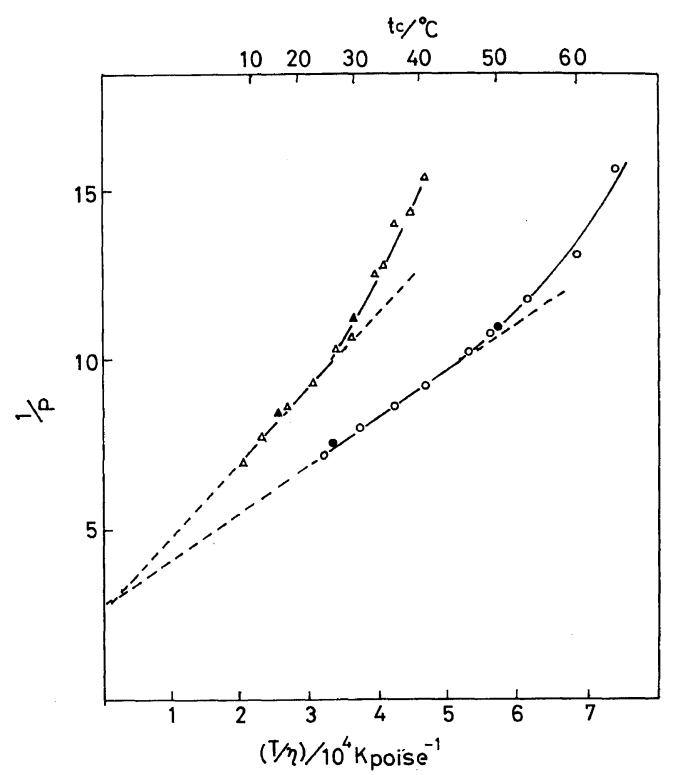

Figure 3. Effect of temperature on the polarization of amylose $(O, \boldsymbol{O})$ and dextran $(\triangle, \boldsymbol{\Delta})$ conjugates. The temperature was increased $(O, \triangle)$ and subsequently decreased $(\boldsymbol{O}, \boldsymbol{\Delta})$. and $3.0 \mathrm{~ns}$ by application of eq 3 . Each value is substantially smaller than that found when $T / \eta$ is varied by sucrose addition at $25^{\circ} \mathrm{C}$. This indicates the presence of temperature-dependent rotations and suggests that some internal flexibility of polysaccharide chain probably increases with temperature. Two kinds of internal rotations should be considered. One is the above-mentioned rotation of the dye around the covalent bond linking to the polymer chain. The other is thermally activated rotation about the glycoside linkage. It is difficult to

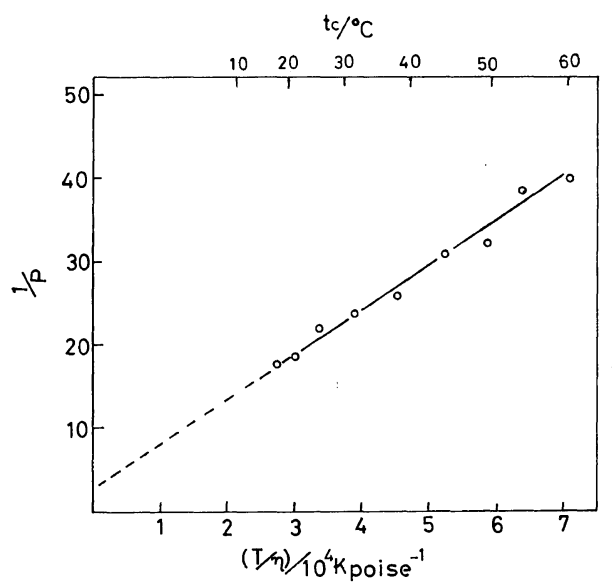

Figure 4. Effect of temperature on the polarization of $\beta$-cyclodextrin conjugate.

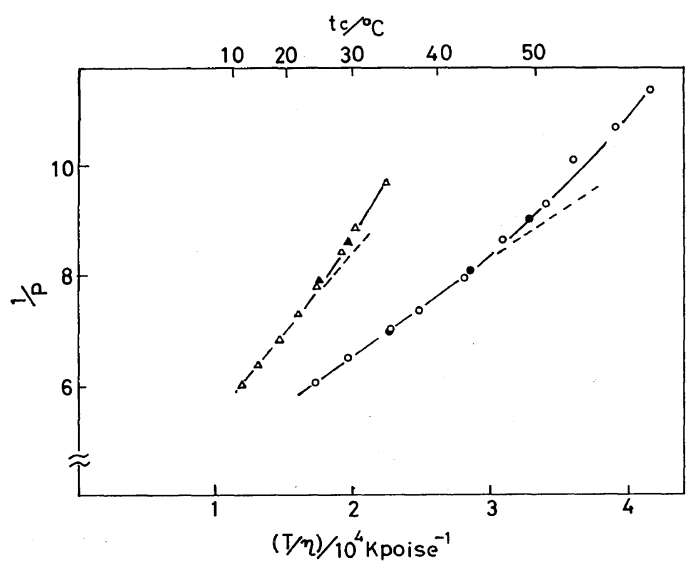

Figure 5. Effect of temperature on the polarization of amylose $(O, \boldsymbol{O})$ and dextran $(\triangle, \boldsymbol{\Delta})$ conjugates in aqueous sucrose solution. Concentration of sucrose, $20 \%(\mathrm{w} / \mathrm{w})$. The temperature increased $(\bigcirc, \triangle)$ and subsequently decreased $(\boldsymbol{O}, \boldsymbol{\Delta})$. 
estimate the contribution to $P$ from these rotations separately. But it is considered that departure from linearity on a Perrin plot is at least attributable to an accelerated loosening of the internal structure of these polymer chains, since departure is not observed for the $\beta$-cyclodextrin conjugate at temperatures ranging from 20 to $60^{\circ} \mathrm{C}$ (Figure 4). $\beta$ Cyclodextrin is a cyclic rigid molecule, so that the conformation of this molecule probably does not change with temperature.

Figure 5 shows Perrin plots for amylose and dextran conjugates when the temperature is changed in the presence of sucrose. A change from a straight line to an upward curve was noted and is shown in Figure 3. $T_{\mathrm{t}}$ is unaffected by the presence of sucrose as shown in Table III. Accordingly, it may be said that the occurrence of this transition depends only on the temperature. The transition temperature for the amylose conjugate is higher than that for the dextran conjugate. Therefore, it may be concluded that the internal structure of amylose is more stable than that of dextran.

\section{DISCUSSION}

A flexible polymer chain does not possess a fixed conformation in solution. Due to thermal motion of the chain segments, the overall conformation of the molecule varies continuously. The extent and rate of such segmental motion are correlated to "dynamic flexibility" of the polymer chain. ${ }^{26}$ The value of the rotational relaxation time thus reflects the flexibility of the polymer. Calculations based on potential energies of non-bonded interaction showed that $\alpha$ 1,6-linked polysaccharide is more flexible than $\alpha$ 1,4-linked polysaccharide. ${ }^{12,13}$ In harmony with this theoretical prediction, the relaxation times obtained in this study indicate that the micro-Brownian motion is more intense in dextran than in amylose.

The results shown in Figures 3 and 5 suggest that the amylose and dextran molecules become more flexible and less ordered with increasing temperature above the transition temperature. The ordered structure of amylose is some type of "helical conformation," which is thought to change to a randomcoil conformation when the temperature is raised. The departure from the Perrin equation of high temperature data in Figures 3 and 5 may be attributed to this conformational change. However, the absence of noticeable temperature dependence in intrinsic viscosity and optical rotation of amylose solutions has been reported by some workers. ${ }^{27-29}$ These results indicate that in the temperature range examined, the molecular chain of amylose doesn't undergo a conformational change analogous to the helix-random coil transition in polypeptides. This discrepancy may be explained by assuming that the conformation of amylose is not in a complete random-coil at high temperatures nor in complete helix at low temperatures. The conformational change with temperature would not be so definite as to prevent a detectable change from occurring in viscosity and optical rotatory power. On the other hand, the fluorescence polarization measurement has been shown to be very sensitive to conformational changes in the short range of the polymer chain since this measurement offers characteristic information on the segmental motion about the attached dye. ${ }^{30,31}$

We conclude that the molecular chains of amylose and dextran do not undergo any great conformational change with temperature, but their conformations change gradually and molecular flexibility increases with increasing temperature above the transition temperature. We also conclude that amylose and dextran below the transition temperature have ordered conformations to some extent. ${ }^{32}$

Acknowledgements. The authors should like to thank Professor Y. Nishijima of Kyoto University for permission to use the nanosecond fluorometer and also thank Dr. S. Ito for his assistance in performing the fluorescence lifetime measurements. Appreciation is due to Associate Professor M. Yamamoto of Kyoto University for his useful comments and criticism.

\section{REFERENCES}

1. G. Weber, Adv. Protein Chem., 8, 415 (1958).

2. Y. Nishijima, J. Polym. Sci., C, 31, 353 (1970).

3. Ph. Wahl, J. Polym. Sci., 29, 375 (1958).

4. M. Frey, Ph. Wahl, and H. Benoit, J. Chim. Phys., 61, 1005 (1964).

5. Y. Nishijima, A. Teramoto, M. Yamamoto, and S. Hiratsuka, J. Polym. Sci., A-2, 5, 23 (1967).

6. T. Teramoto, M. Morimoto, and Y. Nishijima, $J$. Polym. Sci., A-1, 5, 1021 (1967).

7. D. Biddle and T. Nordström, Arkiv Kemi, 32, 359 (1970).

8. E. V. Anufrieva, M. V. Volkenstein, Yu. Ya. Gotlib, 
Polysaccharide Study by the Fluorescence Method. I.

M. G. Krakovyak, S. S. Skorokhodov, and T. V. Sheveleva, Dokl. Akad. Nauk SSSR, 194, 1108 (1970).

9. L. Monnerie and S. Gorin, J. Polym. Sci., C, 30, 99 (1970).

10. B. Valeur and L. Monnerie, J. Polym. Sci., Polym. Phys. Ed., 14, 11 (1976).

11. D. A. Rees, MTR Int. Rev. Sci., Org. Chem. Ser. One, 7, 251 (1973).

12. D. A. Rees and W. E. Scott, J. Chem. Soc., B, 496 (1971).

13. I. Tvaroska, S. Perez, and R. H. Marchessault, Carbohydr. Res., 61, 97 (1978).

14. T. Kuge and S. Ono, Bull. Chem. Soc. Jpn., 34, 1264 (1961).

15. M. Fujii, K. Honda, and H. Fujita, Biopolymers, 12, 1177 (1973).

16. K. Takeo, Y. Kondo, and T. Kuge, Agric. Biol. Chem., 34, 955 (1970).

17. A. N. De Belder and K. Granath., Carbohydr. Res., 30, 375 (1973).

18. S. Nishimoto and Y. Nishijima, Ann. Rep. Res. Inst. Chem. Fibers, Jpn., 32 (1975).

19. R. F. Chen and R. L. Bowman, Science, 147, 729
(1965).

20. G. Weber, Biochem. J., 51, 145 (1952).

21. "International Critical Tables," Vol. V, E. W. Washburn, Ed., McGraw-Hill, 1929, p 23.

22. "Kagaku Binran," Vol. 1 (II), The Chemical Society of Japan, Ed., Maruzen Co., Tokyo, 1966, Sections $5-10$.

23. Ph. Wahl and G. Weber, J. Mol. Biol., 30, 371 (1967).

24. Y. Y. Gottlieb and Ph. Wahl, J. Chim. Phys., 60, 849 (1963).

25. J. Riseman and J. G. Kirkwood, J. Chem. Phys., 17, 442 (1949).

26. K. Shimada and M. Szwarc. J. Am. Chem. Soc., 99, 3313 (1975).

27. J. M. G. Cowie, Makromol. Chem., 53, 13 (1962).

28. W. Burchard, Makromol. Chem., 64, 110 (1963).

29. F. R. Dintzis and R. Tobin, Biopolymers, 7, 581 (1969).

30. T. G. Gill III, Biopolymers, 3, 43 (1965).

31. T. G. Gill III, C. T. Ladoulis, H. W. Kunz, and M. F. King, Biochemistry, 11, 2644 (1972).

32. R. C. Jordan, D. A. Brant, and A. Cesáro, Biopolymers, 17, 2617 (1978). 